\title{
A Digitally Driven Full Mouth Rehabilitation Protocol of a Patient with Hypoplastic Amelogenesis Imperfecta
}

\author{
Simone Abhay Shah ${ }^{1}$, Deepak Nallaswamy² \\ ${ }^{1}$ Department of Prosthodontics, Saveetha Dental College, Chennai, Tamil Nadu, India. \\ ${ }^{2}$ Department of Prosthodontics, Saveetha Dental College, Chennai, Tamil Nadu, India.
}

\section{PRESENTATION OF CASE}

Amelogenesis imperfecta is a developmental disorder which affects enamel. This developmental defect leads to weakening of the enamel leading to an overall discolouration, pitting erosion of the enamel, white hypoplastic spots and multiple carious lesions. A 23-year-old patient came to the Department of Prosthodontics with a chief complaint of aesthetic correction of her teeth. She requested an immediate solution to her problem. She was diagnosed as a case of Hypoplastic Amelogenesis Imperfecta. She showed a strong familial history for this genetic disorder. A digitally driven protocol for full mouth rehabilitation was planned for her to facilitate ease and precision with faster results in a short period of time. Digitally milled all ceramic restorations were planned for marginal accuracy and final results. This hypoplastic variety of AI showed the maximum longevity of dental restorations compared to the other types. This case report elaborates on a combination of conventional and digital rehabilitation of a patient with a hypoplastic type of Amelogenesis Imperfecta.

Amelogenesis Imperfecta is a rare hereditary disorder which causes structural abnormalities in the enamel. ${ }^{1}$ It is a sex linked genetic disorder with either maternal or paternal descent.1,2 There are 3 forms of Amelogenesis Imperfecta - Hypoplastic, Hypocalcific, and Hypomaturation. ${ }^{3}$ The characteristics of this disorder are the loss of tooth structure especially enamel, weakened enamel lead to pitting erosion, yellowish brown discolouration, tendency for multiple carious lesions, dentinal hypersensitivity. 4 This can also lead to the excessive and faster wear of enamel at a young age leading to loss of vertical dimension. ${ }^{5}$ The paternal or maternal family history gives the children a $50 \%$ chance of getting this disorder.

The severity of this disorder varies according to its different types. The most commonly occurring Amelogenesis Imperfecta is the Hypoplastic type. ${ }^{6}$ Around $61 \%$ of the patients have Hypoplastic type of AI. There are multiple variations also seen in this type of Amelogenesis Imperfecta. ${ }^{7}$ This type has the maximum longevity of the dental restorations compared to the other types of Amelogenesis Imperfecta. ${ }^{8}$ Patients with this disorder have a very low morale and confidence in the society. ${ }^{9}$ This leads to social awkwardness and inability to openly smile or laugh. These patients must be handled with utmost care and emotions. ${ }^{10}$ In this case report, we are going to explain a full mouth rehabilitation protocol with a combination of conventional and digital techniques to achieve a stable result functionally and aesthetically.

\section{Extra-Oral Examination}

The profile of the patient showed a mild proclination of the maxillary anterior teeth with multiple rotations of the teeth and absence of a gummy smile. The incisal line was non-coherent with the lower lip line. This as well as the discoloured teeth lead to an un-aesthetic smile. There was a mild drooping of the corners of the lips.

\author{
Corresponding Author: \\ Dr. Simone Shah, \\ Department of Prosthodontics, \\ Saveetha Dental College, \\ Chennai - 600077, Tamil Nadu, \\ India. \\ E-mail: simoneshah2293@gmail.com
}

DOI: 10.14260/jemds/2020/622

How to Cite This Article:

Shah SA, Nallaswamy D. A digitally driven full mouth rehabilitation protocol of a patient with hypoplastic amelogenesis imperfecta. J Evolution Med Dent Sci 2020;9(38):2852-2856,

$10.14260 / \mathrm{jemds} / 2020 / 622$

Submission 17-06-2020,

Peer Review 12-08-2020,

Acceptance 19-08-2020,

Published 21-09-2020.

Copyright (C) 2020 Simone Abhay Shah et al. This is an open access article distributed under Creative Commons Attribution License [Attribution 4.0 International (CC $B Y 4.0)]$ 

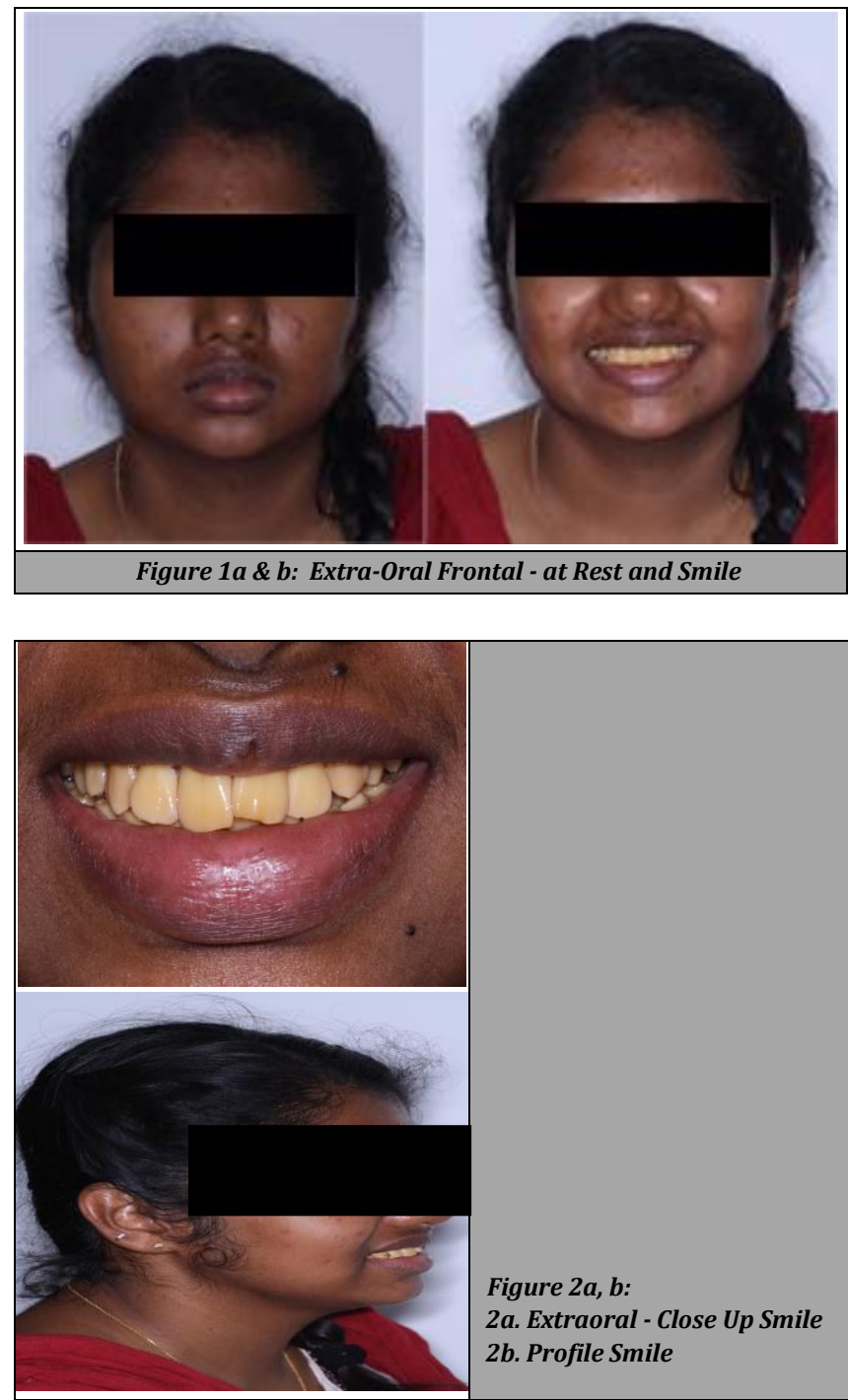

Figure $2 a, b$ :

2a. Extraoral - Close Up Smile 2b. Profile Smile

\section{Intra-Oral Examination}

Yellowish brown discolouration of all the teeth. Hypoplastic white spots seen on the cervical aspect of the teeth. The occlusion was Class 2 div 1 subdivision with posterior bilateral crossbite. The patient had a mutually protected occlusion with protrusive and laterotrusive contacts at the incisor. (Figure 3)

Multiple carious lesions with respect to $16,17,18,26,27,28,37,36,47,46$. Multiple rotations in maxillary and mandibular anterior segments.

\section{APPOINTMENT 1}

\section{Clinical}

1. Patient's extraoral and intraoral photographic records were taken. Patient consent was obtained for the above treatment plan.

2. Maxillary and mandibular diagnostic impressions were made and 2 sets of casts were poured.

3. Assessment of the freeway space, the vertical dimension at rest and occlusion were made. The freeway space was $3 \mathrm{~mm}$ which is in the normal range.

4. An Orthopantomogram (OPG) was taken to evaluate the enamel and dentinal condition. Enamel wear was observed with multiple carious lesions. There was no horizontal and vertical bone loss suggesting a stable healthy periodontium.

5. A Deprogrammer (Lucia Jig) was given to the patient for 30 minutes after which a centric bite was taken at the vertical dimension of occlusion. A Facebow record was made using the UTS 3D Ivoclar Vivadent facebow.
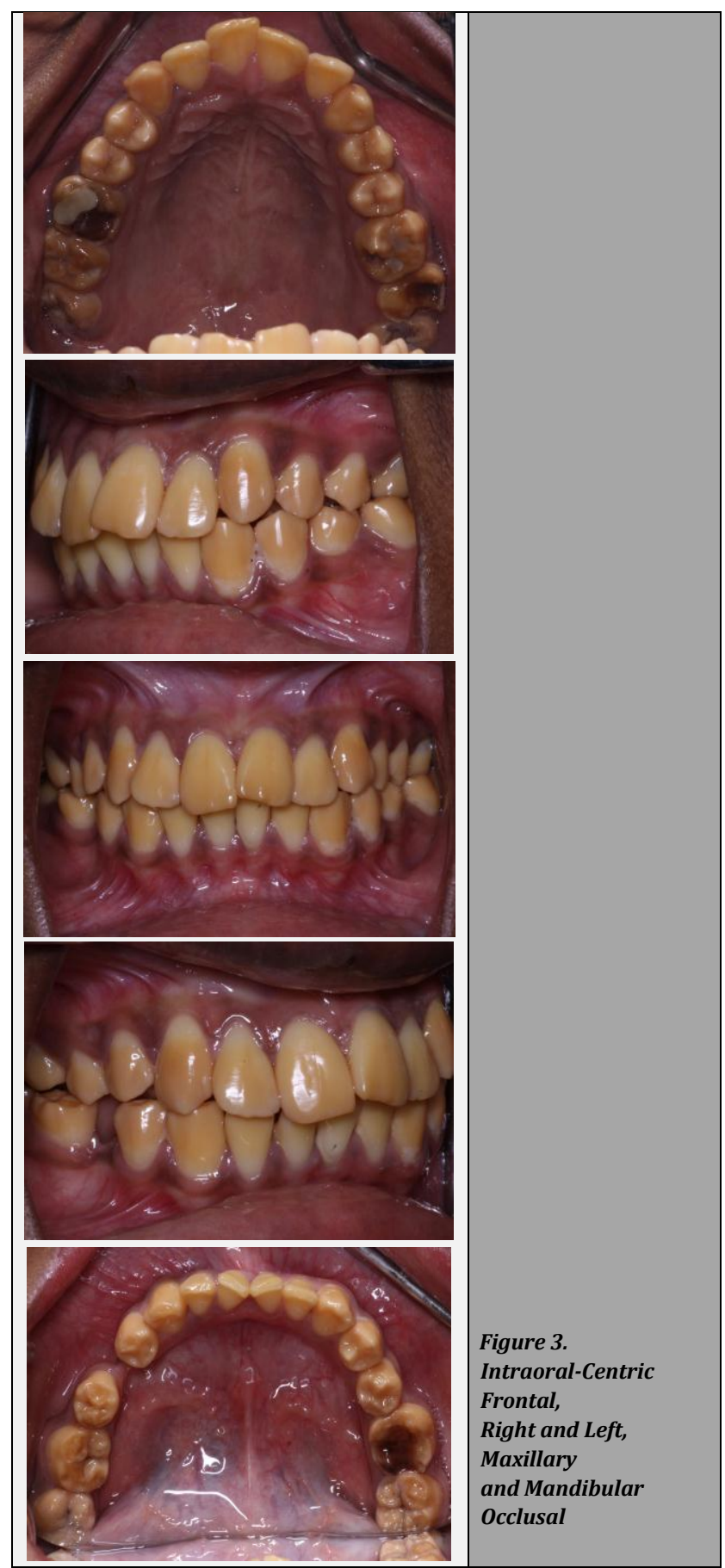

\section{Laboratory}

1. The casts are mounted at this recorded vertical dimension and centric relation on Stratos 300 semi-adjustable articulator.

2. Mock preparations were made on the cast's followed by a digital diagnostic wax up planned on 3 shape TRIOS design studio. 
3. This diagnostic wax was used to fabricate polymethylmethacrylate (PMMA) temporary teeth on the IMES icore CORiTEC 350i machine.

\section{APPOINTMENT 2}

\section{Clinical}

1. Maxillary and mandibular full mouth preparations were done with adequate clearance for a full coverage all ceramic restoration.

2. The PMMA temporary teeth were then retrofitted and relined for precise marginal fit.

3. The occlusion was adjusted to a mutually protected occlusion and the plane of the temporaries were adjusted. These temporaries were given for 6 weeks to make the patient comfortable with the recorded centric and the new set of teeth.

\section{APPOINTMENT 3}

\section{Clinical}

1. Maxillary and mandibular tooth preparations are refined.

2. Double cord packing technique with a 2 stage putty wash technique was used to take final impressions of the maxillary and mandibular arches. 00 (Impregum,3M Germany) and 0 cords (Impregum,3M Germany) were used with Zhermack Putty and Zhermack Elite HD light body material (Addition Silicone).

3. Bilateral bite was recorded using the conventional method for jaw relation record using pattern resin.

4. A facebow record was made using UTS 3D Ivoclar Vivadent.

\section{Laboratory}

1. The final casts were fabricated using die stone and then mounted on the semi adjustable Stratos 300 articulator using the facebow record and the bilateral pattern resin bite.

2. The casts and the bite was then scanned in the Medit T500 3D Lab scanner.

3. The scans are then Imported onto the 3shape TRIOS design studio software for fabrication of the final restoration

4. Full mouth individual Zirconia copings are fabricated digitally leading to an extremely accurate fit.

5. The designed Zirconia copings are then soft milled in the IMES icore 350i CORiTEC machine.

\section{APPOINTMENT 4}

\section{Clinical}

1. A trial of the Zirconia copings fabricated was done to check the marginal fit.

2. An orthopantomogram (OPG) was taken to confirm the accurate marginal fit.

\section{Laboratory}

1. Zirconia copings are then hand layering with ceramic.

\section{APPOINTMENT 5}

\section{Clinical}

1. A Ceramic trial was done prior to final glazing

2. The smile line, phonetics, aesthetics, function and occlusion were evaluated to establish an accurate functional and aesthetic restoration.

3. After establishing the accurate final outcome and once the patient is satisfied the ceramic trial was sent for glazing

4. The final restoration was cemented using glass ionomer restoration.

5. Patient was then asked to follow strict oral hygiene protocols and asked to report every 3 months for a routine follow up. The patient has been following up since a year now.

6. Occlusal Splint was given to the patient.
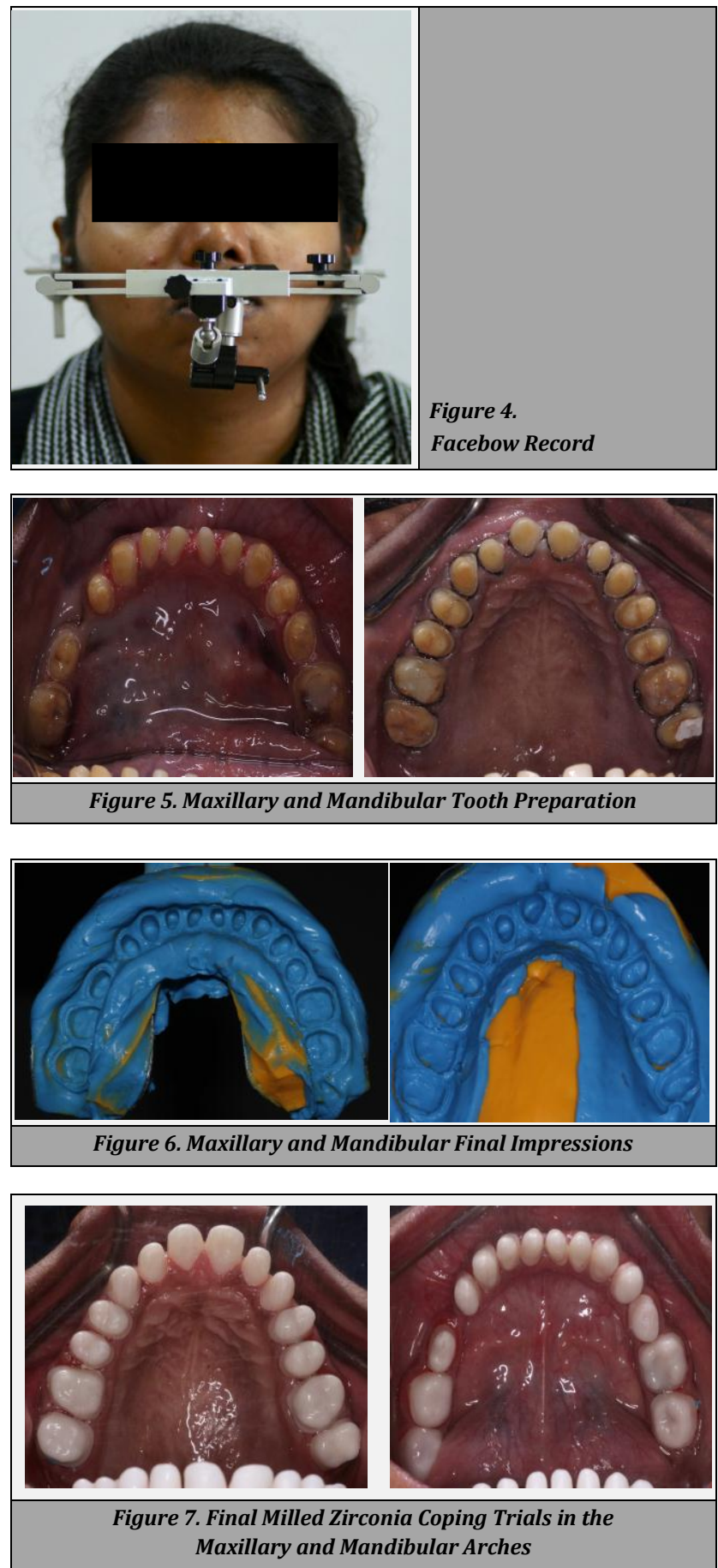

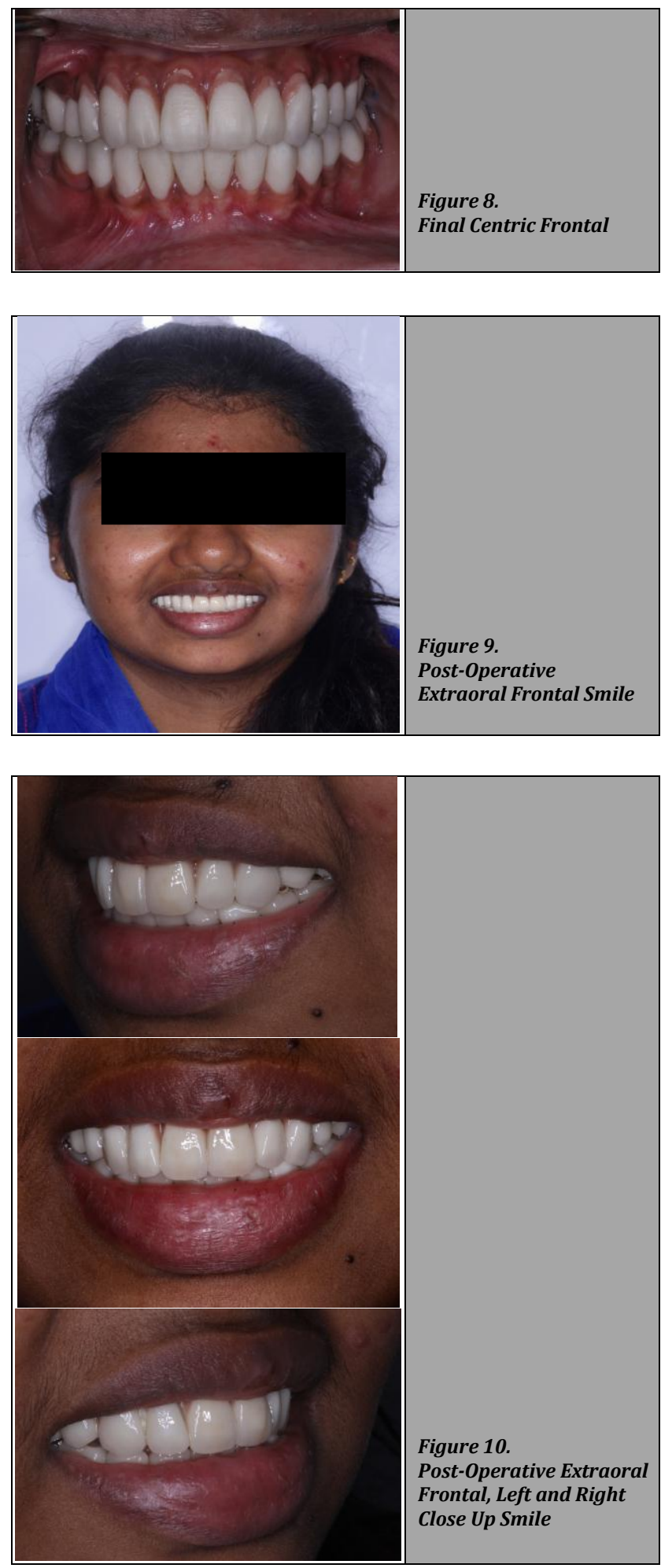

\section{DISCUSSION}

Zirconium dioxide based restoration used had excellent mechanical properties, was biocompatible and was resistant to bacterial adhesion. ${ }^{11}$ Zirconium dioxide is highly opaque, thus this helps in masking the discolouration of the underlying enamel in patients with amelogenesis imperfecta. ${ }^{12}$ Thus, using a Zirconia layered all ceramic restoration was a good restorative material for this case.
Defective enamel formation seen in cases of amelogenesis imperfecta, in such cases it is preferable to bond the restoration onto the dentin. ${ }^{13}$ Ideal restorations would be porcelain fused to metal or all ceramic restorations. ${ }^{8}$

Canine guided occlusion was established to achieve posterior disocclusion on protrusive and laterotrusive movements. ${ }^{14}$ This occlusion has been proven to be stable over a longer period of time. ${ }^{15}$

The fabrication of the PMMA temporaries and the zirconia copings were done digitally. ${ }^{16}$ This established accurate margins and thus reduced the marginal discrepancy. ${ }^{17}$ CAD / CAM enabled visualization of the tooth preparation margins accurately to reproduce snugly fitting prosthesis with ideal cement space. ${ }^{17}$

The patient related outcome measures like OHIP - 14 questionnaire and Rosenberg self - evaluation scale were evaluated before and after the treatment. ${ }^{18}$ The patient's self esteem post the treatment improved drastically. ${ }^{17,19}$

The final outcome leads to improved esthetics and function for the patient. This improved the patient satisfaction and improved the patient's social life and emotional quotient. Establishing a positive treatment outcome enhances the value of the treatment and leads to an immense personal and professional satisfaction.

\section{CONCLUSIONS}

Optimal diagnosis and planning with a clearly established protocol leads to satisfactory results always.

Financial or Other Competing Interests: None.

\section{REFERENCES}

[1] Witkop CJ. Amelogenesis imperfecta, dentinogenesis imperfecta and dentin dysplasia revisited: problems in classification. J Oral Pathol 1988;17(9-10):547-53.

[2] Ergun G, Ataol AS. An interdisciplinary approach for hypoplastic amelogenesis imperfecta: a case report. Open Dent J 2018;12:466-75.

[3] Fischman SL, Fischman BC. Hypoplastic amelogenesis imperfecta: report of case. The Journal of the American Dental Association 1967;75(4):929-31.

[4] Darling AI. Some observations on amelogenesis imperfecta and calcification of the dental enamel. Proc $\mathrm{R}$ Soc Med 1956;49(10):759-65.

[5] de Souza JF, Fragelli CMB, Paschoal MAB, et al. Noninvasive and multidisciplinary approach to the functional and esthetic rehabilitation of amelogenesis imperfecta: a pediatric case report. Case Rep Dent 2014;2014:1-5

[6] Crawford PJM, Aldred M, Bloch-Zupan A. Amelogenesis imperfecta. Orphanet J Rare Dis 2007;2:17.

[7] Basnet BB, Parajuli PK, Singh RK, et al. A case report of full-mouth rehabilitation of amelogenesis imperfecta with improved oral health impact profile. International Journal of Dental Sciences and Research 2014;2(6):133-6. 
[8] Husein A, Al Harun KAM, Ghazali L, et al. Full mouth rehabilitation in a patient with amelogenesis imperfecta: treatment for aesthetic and functional improvement. British Journal of Medicine and Medical Research 2015;8(9):806-10.

[9] Canger EM, Çelenk P, Yenísey M, et al. Amelogenesis imperfecta, hypoplastic type associated with some dental abnormalities: a case report. Braz Dent J 2010;21(2):1704.

[10] Simmer JP, Hu JC. Dental enamel formation and its impact on clinical dentistry. J Dent Educ 2001;65(9):896-905.

[11] Green DJ. Transformation toughening of ceramics. CRC Press 2018: p. 240.

[12] Tuncel İ, Turp I, Üşümez A. Evaluation of translucency of monolithic zirconia and framework zirconia materials. J Adv Prosthodont 2016;8(3):181-6.

[13] Arshad M, Shirani G, Mahgoli HA, et al. Rehabilitation of a patient with amelogenesis imperfecta and severe open bite: a multidisciplinary approach. Clin Case Rep 2019;7(2):275-83.
[14] Schierz O, Reissmann D. Influence of guidance concept in complete dentures on oral health related quality of life Canine guidance vs. bilateral balanced occlusion. J Prosthodont Res 2016;60(4):315-20.

[15] Heydecke G, Akkad AS, Wolkewitz M, et al. Patient ratings of chewing ability from a randomised crossover trial: lingualised vs. first premolar/canine-guided occlusion for complete dentures. Gerodontology 2007;24(2):77-86.

[16] Masri R, Driscoll CF. Clinical applications of digital dental technology. John Wiley \& Sons 2015: p. 272.

[17] Asaad RS. Marginal accuracy of CAD/CAM fabricated monolithic all-ceramic crowns as a function of digital scanning techniques. Egyptian Dental Journal 2019;65(2):1925-38.

[18] Kireilytė M, Masiliūnaitė V, Belickienė V, et al. Testing of Lithuanian version of the Oral Health Impact Profile-14 among older adults. A pilot study. Stomatologija 2019;21(3):67-71.

[19] McHale ST. The Rosenberg self-esteem scale and its correlates among Russian and American Youth. Doctoral dissertation, University of Maryland at College Park 1994: p. 88. 